\title{
An Automated Graphical User Interface based System for the Extraction of Retinal Blood Vessels using Kirsch's Template
}

\author{
Joshita Majumdar \\ Dept. of Electronics \& Communication \\ IEM, WBUT \\ Kolkata, India \\ Souvik Tewary \\ Dept. of Bio Medical Engineering \\ NSEC, WBUT \\ Kolkata, India \\ Shreyosi Chakraborty \\ Dept. of Bio Medical Engineering \\ NSEC, WBUT \\ Kolkata, India
}

\author{
Debasish Kundu \\ Principle (ECE) \\ Gobindapur Sapahali Memorial Polytechnic \\ Burdwan, India \\ Sudipta Ghosh \\ Dept. of Electronics \& Communication \\ Bengal Institute of Technology \& Management \\ Shantiniketan, India \\ Sauvik Das Gupta \\ School of Electrical \& Computer Engineering \\ Oklahoma State University \\ OK, USA
}

\begin{abstract}
The assessment of Blood Vessel networks plays an important role in a variety of medical disorders. The diagnosis of Diabetic Retinopathy (DR) and its repercussions including micro aneurysms, haemorrhages, hard exudates and cotton wool spots is one such field. This study aims to develop an automated system for the extraction of blood vessels from retinal images by employing Kirsch's Templates in a MATLAB based Graphical User Interface (GUI). Here, a RGB or Grey image of the retina (Fundus Photography) is used to obtain the traces of blood vessels. We have incorporated a range of Threshold values for the blood vessel extraction which would provide the user with greater flexibility and ease. This paper also deals with the more generalized implementation of various MATLAB functions present in the image processing toolbox of MATLAB to create a basic image processing editor with different features like noise addition and removal, image cropping, resizing \& rotation, histogram adjust, separately viewing the red, green and blue components of a colour image along with brightness control, that are used in a basic image editor. We have combined both Kirsch's Template and various MATLAB Algorithms to obtain enhanced images which would allow the ophthalmologist to edit and intensify the images as per his/her requirement for diagnosis. Even a non technical person can manage to identify severe discrepancies because of its user friendly appearance. The GUI contains very commonly used English Language viz. Load, Colour Contrast Panel, Image Clarity etc that can be very easily understood. It is an attempt to incorporate maximum number of image processing techniques under one GUI to obtain higher performance. Also it would provide a cost effective solution towards obtaining high definition and resolution images of blood vessel extracted Retina in economically backward regions where costly machine like OCT (Optical Coherence Tomography), MRI (Magnetic Resonance Imaging) are not available. Hence an early detection of irregularity will be possible especially in rural areas.
\end{abstract}

Keywords-Blood Vessel Extraction; Retinal Images; Kirsch's Templates; Image Processing; Graphical User Interface

\section{INTRODUCTION}

The vascular network is an essential anatomical structure in the human retina. An analysis of the retinal vasculature may lead to the diagnosis of various abnormalities such as haemorrhages, micro aneurysms etc. thus, an automated system for retinal blood vessel extraction is a preliminary step in the development of a computer-assisted diagnostic system for ophthalmic anomalies.

The extraction of the human eye vasculature from retinal images involves the essential tool of edge detection. According to Muthukrishnan \& Radha [1], the classical methods of edge detection operates on convolving the image through an operator. Yin et al. [2] proposed a probabilistic tracking method to detect blood vessels in retinal images. They categorized the task of vessel extraction into two main groups: Pixel-based methods and tracking methods.

According to Zhang et al. [3], matched filter is a simple yet effective method for vessel extraction. They also proposed an extension to the matched filter approach to detect retinal blood vessels that significantly reduce the false detections produced by the original matched filter. Esmaeili et al. [4], presented an efficient algorithm for automatic extraction of blood vessels that comprises the following four steps: (i) Curvelet-based contrast enhancement, (ii) Matched filtering, (iii) Curvelet based edge-extraction and (iv) Length filtering. In their study, the enhanced image is first reconstructed from the modified curvelet co-efficient followed by match filtering to intensify the blood vessels along with the implementations 
of curvelet transform to segment vessels from its background. Finally, they have used length filtering to remove the misclassified pixels. Their experimental results have been evaluated on DRIVE database (Niemaiger \& van Ginneken, [5]). The images on which we worked on have been collected from a publicly available DRIVE database and from the Regional Institute of Ophthalmology, Medical College, Kolkata [6].

The next section describes the hardware \& software platforms of our system. Section III presents the methodology we proposed for blood vessel extraction. Sections IV \& V deal with the relevant discussions and Future Prospects respectively. The conclusion of the paper is presented in Section VI.

\section{HARDWARE AND SOFTWARE PLATFORMS}

Minimum Hardware requirements:

- Dual core processor

- RAM: 4 GB

- Windows XP/7/8

- $256 \mathrm{MB}$ Graphics card(to enhance the performance of the GUI)

Our study has been conducted using:

- Laptop (SONY VAIO VPCEH16EN)

- Windows 7

- Intel ${ }^{\circledR}$ Core $^{\mathrm{TM}}$ i3-2310M Processor $2.10 \mathrm{GHz}$

- $\quad$ RAM : 4 GB

- 256 MB nVIDIA GEFORCE ${ }^{\circledR}$ with CUDA.

The software used to develop the GUI is MATLAB which is a multi-paradigm numerical computing environment and $4^{\text {th }}$ Gen. Programming language.

\section{METHODOLOGY}

The complete procedure of extracting the blood vessels from the coloured retinal image consist of three steps, beginning with fetching the input image from the system or the camera, then converting the RGB image into a gray scale image and finally using the kirsch's templates to detect the edges of the blood vessels. Fig. 1 illustrates the flow diagram of the proposed method for blood vessel extraction. a) Fetching the image: Two separate push buttons on the GUI facilitate the input of an image. One allows the user to browse an image from the system hard drive or any other external drives and the other allows the user to capture an image using any camera attached to the computer. The image that has been loaded is displayed on the first axis positioned on the top left corner of the GUI.

b) Gray Conversion: The panel 'Colour format' in the GUI contains a radio button 'GRAY Scale' that enables the user to convert the RGB retinal image to gray scale having pixel values ranging from 0 to 255 with just one mouse click.

c) Extraction of Blood Vessels using Kirsch's Templates: The Kirsch operator or Kirsch compass kernel is a non-linear edge detection that finds the maximum edge strength in a few pre determined directions. The 'VESSEL EXTRACTION' panel contains a popup menu with a number of threshold values (within a range of 4.6 to 9.0) for blood vessel extraction. The Kirsch operator can adjust the related threshold value automatically due to the image characteristics. The operator takes a single kernel mask and rotates it in $45^{\circ}$ increments through all eight compass directions: North, North-West, West, South-West, South, South-East, East and North-East as shown below:

$$
M 1=\left(\begin{array}{ccc}
5 & 5 & 5 \\
-3 & 0 & -3 \\
-3 & -3 & -3
\end{array}\right) M 2=\left(\begin{array}{ccc}
5 & 5 & -3 \\
5 & 0 & -3 \\
-3 & -3 & -3
\end{array}\right) M 3=\left(\begin{array}{ccc}
5 & -3 & -3 \\
5 & 0 & -3 \\
5 & -3 & -3
\end{array}\right) M 4=\left(\begin{array}{ccc}
-3 & -3 & -3 \\
5 & 0 & -3 \\
5 & 5 & -3
\end{array}\right)
$$

$$
M 5=\left(\begin{array}{ccc}
-3 & -3 & -3 \\
-3 & 0 & -3 \\
5 & 5 & 5
\end{array}\right) M 6=\left(\begin{array}{ccc}
-3 & -3 & -3 \\
-3 & 0 & 5 \\
-3 & 5 & 5
\end{array}\right) M 7=\left(\begin{array}{ccc}
-3 & -3 & 5 \\
-3 & 0 & 5 \\
-3 & -3 & 5
\end{array}\right) M 8=\left(\begin{array}{ccc}
-3 & 5 & 5 \\
-3 & 0 & 5 \\
-3 & -3 & -3
\end{array}\right)
$$

The edge magnitude of the Kirsch operator is calculated as the maximum magnitude across all directions. Except the outermost rows and columns, every pixel along with its eight neighbouring pixels in a given image is convolved with the eight aforementioned templates respectively [7], providing eight outputs for each pixel, the maximum of which is defined as the edge magnitude (Gao et al. [8]. A pixel's Gray value with its eight neighbours is as shown.

\begin{tabular}{|ccc|}
\hline $\mathrm{P}_{0}$ & $\mathrm{P}_{1}$ & $\mathrm{P}_{2}$ \\
$\mathrm{P}_{7}$ & $\mathrm{P}(\mathrm{i}, \mathrm{j})$ & $\mathrm{P}_{3}$ \\
$\mathrm{P}_{6}$ & $\mathrm{P}_{5}$ & $\mathrm{P}_{4}$ \\
\hline
\end{tabular}

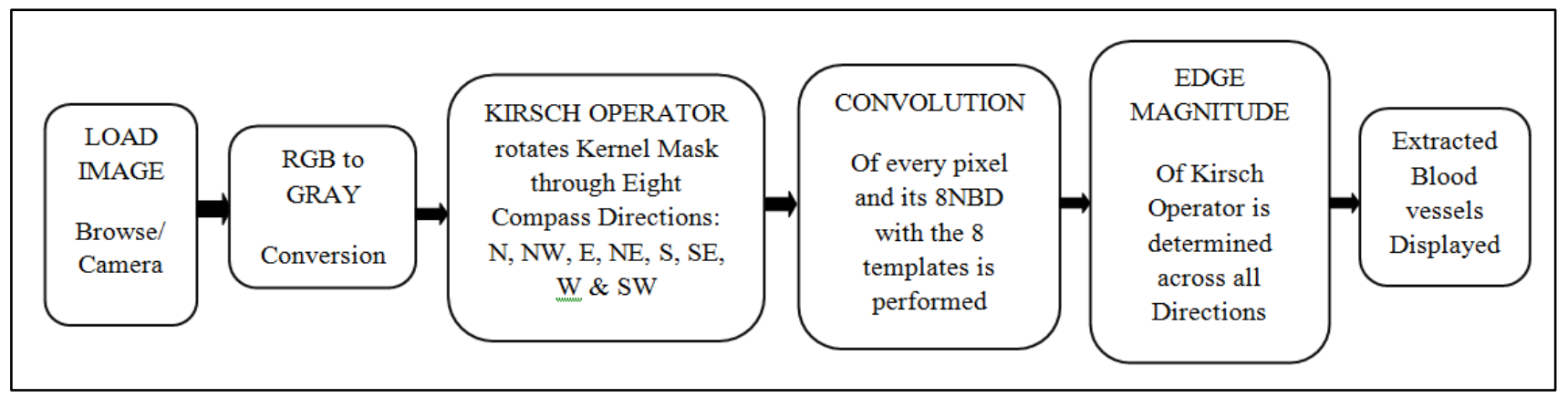

Fig. 1. Proposed Overall Method for Blood Vessel Extraction 
The direction of edge is defined by the related mask that produces the maximum magnitude. The general output of edge detection through Kirsch's Templates is an image containing Gray level pixels of value 0 or 255 . The value 0 indicates a black pixel and the value 255 indicates a white pixel. The edge information of the target pixel is checked by determining the brightness levels of the neighbouring pixels [9]. In case no major difference in the brightness level is found, the possibility of the pixel being a part of an edge is ruled out.

Kirsch's Template can set and reset the threshold values to obtain most suitable edge of images. It works well for images having a clear distinction between the foreground and background [10]. Since the retinal blood vessels can be considered as the required foreground information from the background fundus images, Kirsch's algorithm is effectively applicable. Fig. 2 illustrates an original fundus image with the extracted blood vessels.

\section{d) Other Important Tools:}

A fact worth mentioning is that the GUI designed in order to carry out this study on the extraction of blood vessels may also be used to edit and enhance the retinal images for further analysis using the following options.

- Image Addition

- Image Adjust Panel

- Pixel Processing, Image Rotate, Image Complement, Colour Swap

- Image Resize, Image Crop

- Contrast Slider, R G B Sliders

- CMY Panel

- Colour Panel - Gray, B \& W, Pseudo

- Noise Addition, Filter

- Edge Detection

- B \& W Morphological Operations

- Colour Plane Models

$\begin{array}{ll}\circ & \text { Hue } \\ \circ & \text { Saturation } \\ \circ & \text { Value } \\ \circ & \text { HSV }\end{array}$

\section{- Image Save etc \\ ○ Luma}

Some of these features have been discussed below:

1) Image Addition: Image is a simple matrix. Since addition can be performed on matrix, so that can also be applied on images. To do this a function available in MATLAB is used which performs addition of pixel values of first image to the second image. Addition of the image by itself gives a better clarity of the original image. Fig. 3 demonstrates the image addition operation.

2) Image Clarity: Generally for certain images the pixel distribution is not equally spaced or rather are clotted to a particular intensity level, hence, making the image too dull or too bright [11]. For this reason the following histogram adjustment techniques are being used: Image Adiust, Histogram equalization, Adaptive Histogram equalization. Fig 5. Illustrates the histogram equalization feature.

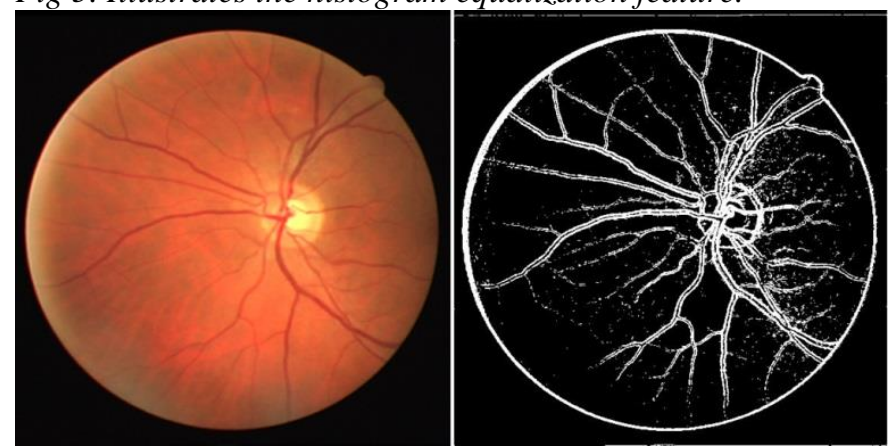

Fig. 2. Extracted Blood Vessels

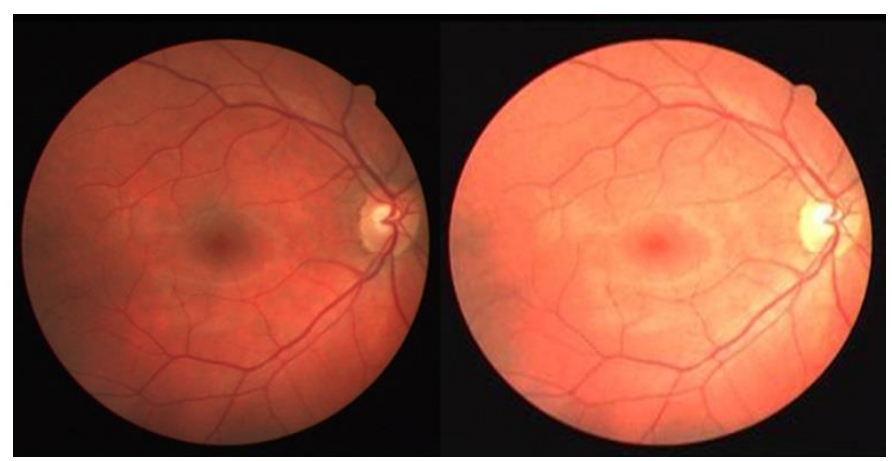

Fig. 3. Demonstration of Addition operation on the image 


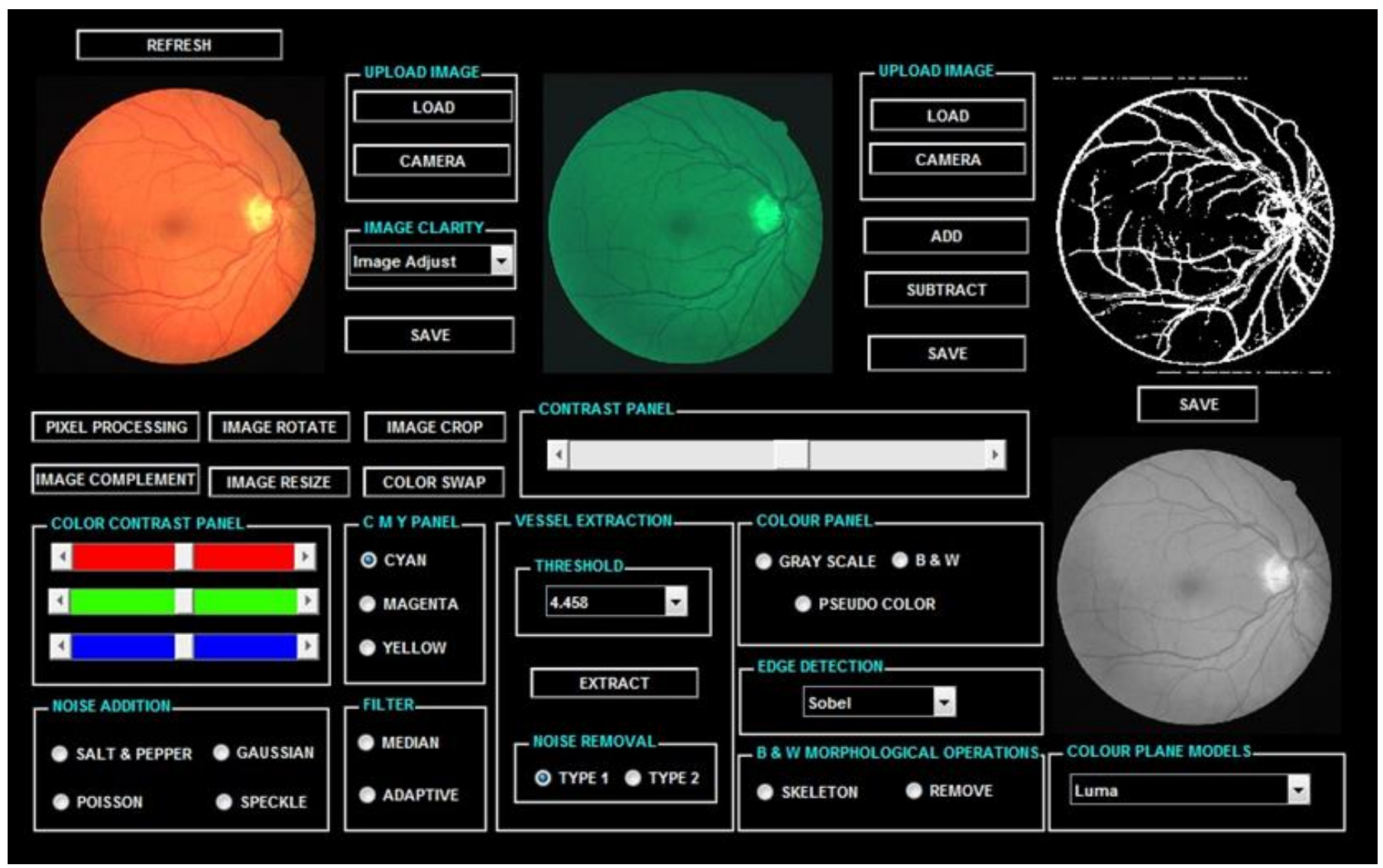

Fig. 4. Complete MATLAB based GUI demonstrating the extraction of Retinal Blood Vessels Using Kirsch's Templates

3) Image Rotate: Image rotation is used to rotate the image to a specified degree and get a different perspective of view.

4) Image Complement: Image complement simply complements the image. Here, the RGB image of the fundus is complemented where each pixel value is subtracted from the maximum pixel value supported by the class and the difference is used as the pixel value in the output image.

5) Image resize: It is being used to resize the actual image to certain multiples.

6) Image crop: It is used to select any particular portion of the whole image.

7) Contrast Panel: This panel is used to apply brightness or contrast to the image. It is varied over a range of 0 to 255 .

8) Colour Contrast Panel: Slider for Red, Green and Blue are added to view the single coloured image in varied brightness (dark and light). It has been mentioned that a colour or RGB image is an overlap of three two dimensional matrices.

9) CMY Panel: This panel is used to view the Cyan, Magenta and Yellow components of the image separately.

10)Colour Panel: The most important feature of this panel is the 'GRAY' radio button that converts an RGB image into a Gray scale image.

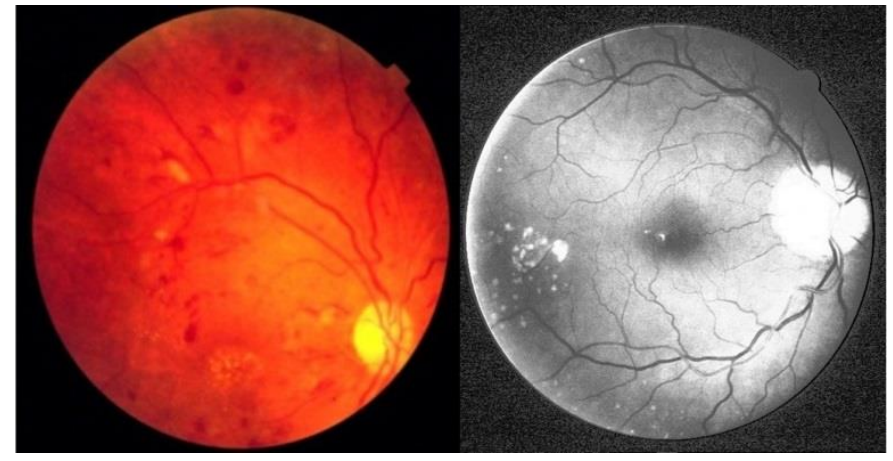

Fig. 5. Image Adjust and Histogram Equalization

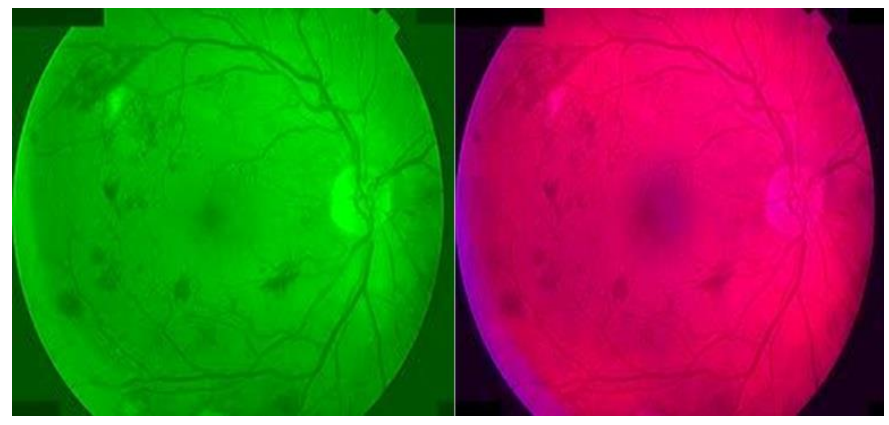

Fig. 6. Green \& Magenta Images in addition with contrast 
11)Noise addition and removal: Various types of noises get added to an image when a snapshot is taken. In order to get rid of these noises various types of filters are used. To illustrate this we have added a noise to an image externally and then applied various filters to get rid of it and evaluated the results. The types of noises available in this GUI are Poisson, Salt \& Pepper, Gaussian and Speckle. Noise removal maybe performed using either a Median filter or an Adaptive filter. Fig. 9. Show the addition and removal of 'salt and pepper noise'.

12)Edge detection: The various types of edge detection techniques are: Sobel, Prewitt, Roberts, LoG, Canny and Zerocross. These options are available for the user to compare the results with those of the Kirsch's Algorithm.

13)Colour Plane Models: In this panel the ophthalmologist would get the opportunity to select any of the following image type which would enable a different cylindrical-coordinate representation of the RGB Colour image:

a) Hue image: Using this option might enable the ophthalmologist to detect further abnormalities of the fundus [12] that are not very evident from the Retinal Blood Vessel Extraction.

b) Saturation image: This option would let the ophthalmologist view the picture of the fundus in a different colour tone as shown in Fig. 8.

c) Hue Saturation Value (HSV) image: The combination of Hue Saturation and Value gives a better outlook of the fundus for detection of abnormalities.

d) Luminosity (luma) image: By this option the ophthalmologist gets the view of the weighted sum of the gamma corrected image of the main RGB image of the fundus.

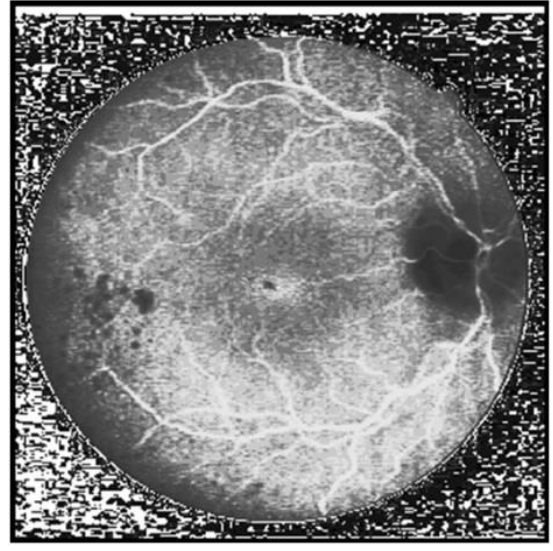

Fig. 7. Hue Image

14)Save: The Save option is provided against the three working axes present in the middle, top right corner and bottom right corner of the GUI for the purpose of saving the edited or worked on images for future reference.

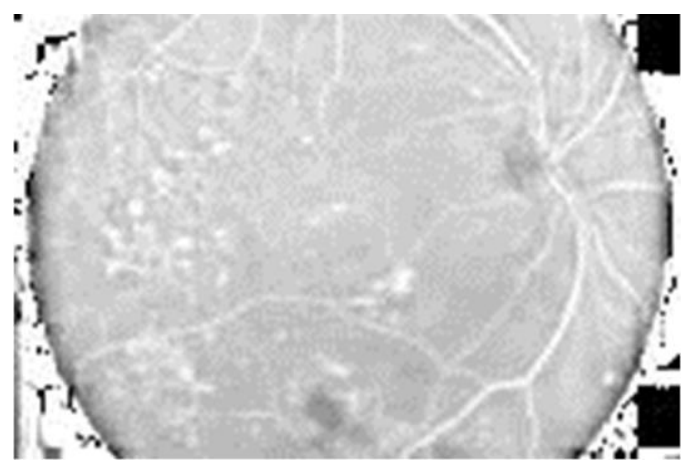

Fig. 8. Saturation Image

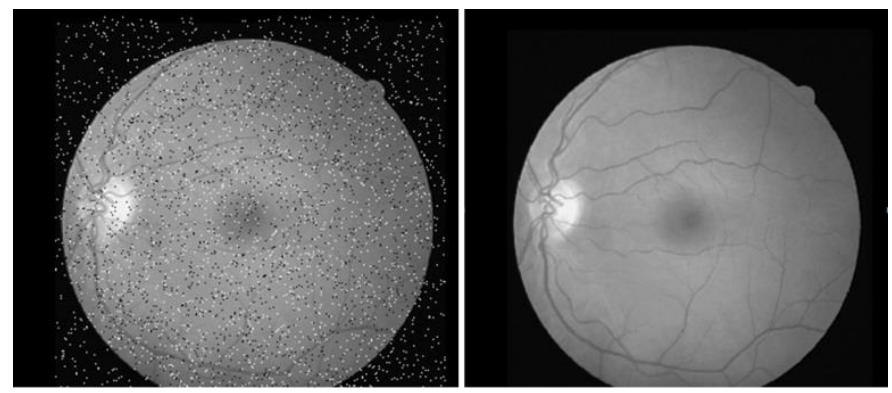

Fig. 9. Demonstration of Addition and Removal of 'Salt \& Pepper' Noise using a Median Filter

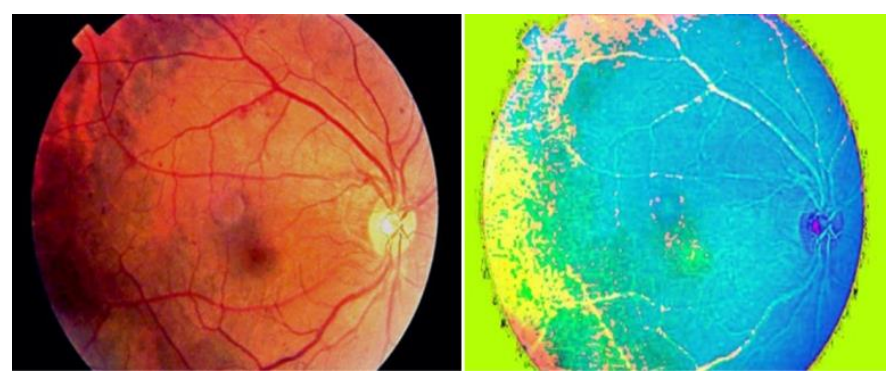

Fig. 10. Demonstration of HSV Image in comparison to the original Fundoscopic Image

\section{DISCUSSIONS}

Diabetic Retinopathy is an important cause of blindness. It is an outcome of prolonged accumulated damage caused to the retinal blood vessels. One percent of global blindness is as a result of Diabetic Retinopathy [13]. We have presented in this paper the retinal blood vessel extraction with the help of Kirsch's Templates and a combination of various MATLAB image processing techniques. The combination of these two techniques provided an optimum result. Table1 depicts the comparison between our Results and other Techniques in the extraction of Blood Vessels.

The interface is presented in a user-friendly manner. Individual Save buttons next to each and every image window helps the user to re-use and re-apply the extraction process on edited images. This process enhances the base image at every stage. 
Our main aim is to provide a cost effective solution to detect Retinal anomalies and provide a better platform. In some cases, minute examination of Diabetic Retinopathy is carried on and analyzed by Fundus Photography and for detailed insight Optical Coherence Tomography is used [17]. Table 2 represents a comparative study between our Graphical User Interface and the present clinical technique for the detection of Retinal diseases.

TABLE I. COMPARISON OF OUR RESUlts WITH OTHER TECHNIQUES FOR BLOOD VESSEL EXTRACTION

\begin{tabular}{|l|}
\hline Raw Photo \\
\hline Pas \\
\hline
\end{tabular}
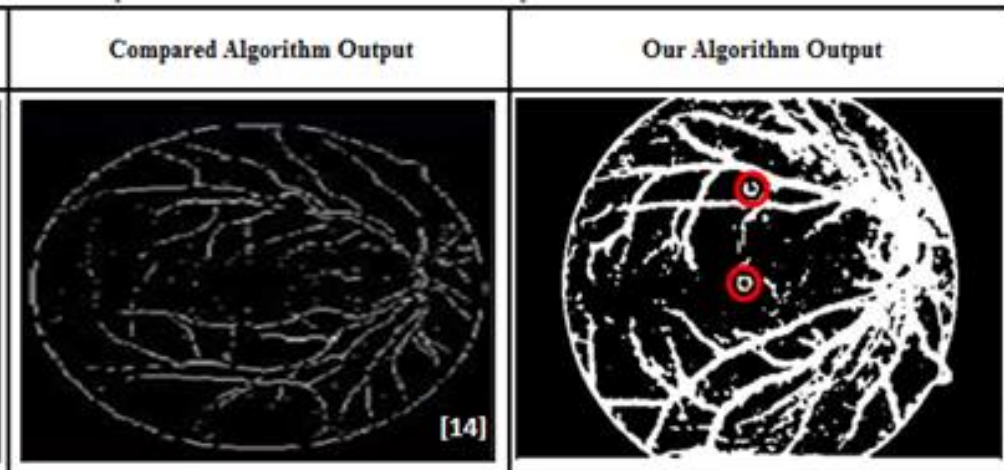

The ones marked in Red
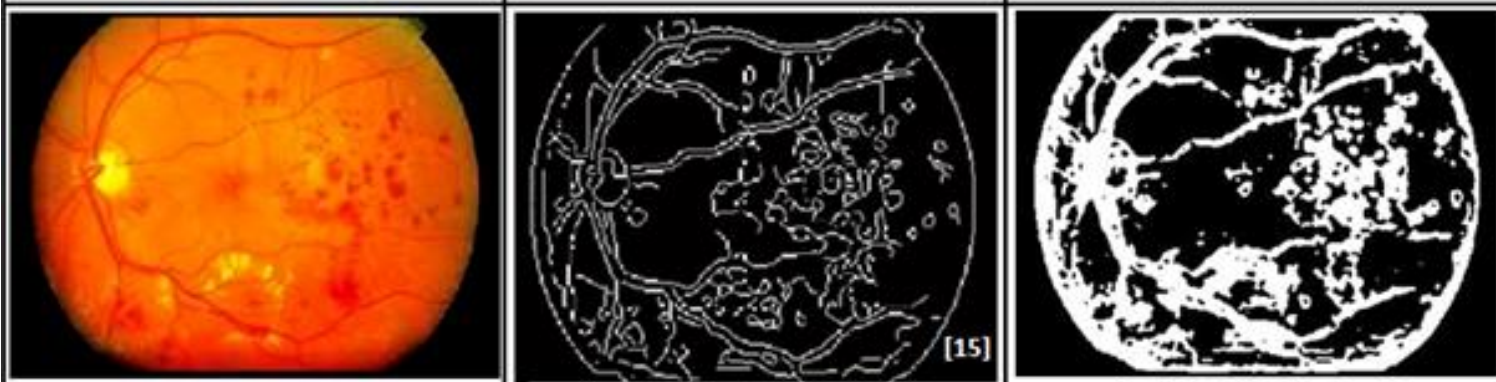

depicts the Lesions.
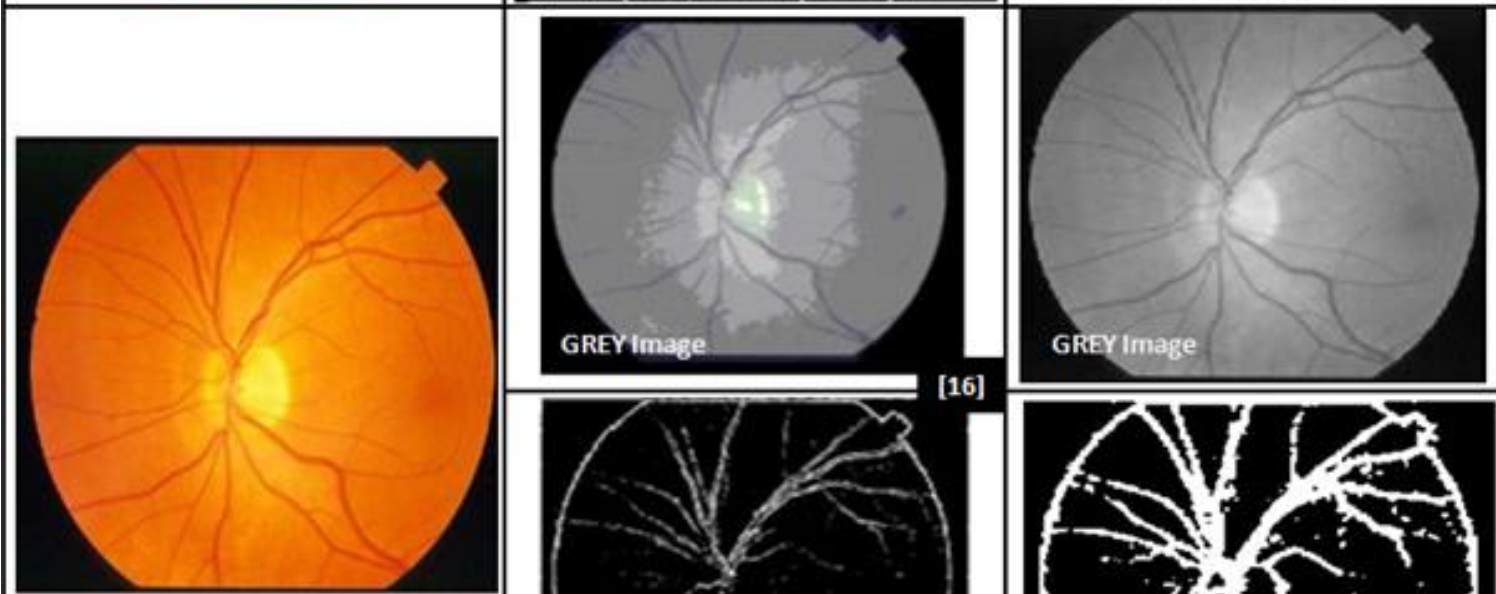

The lesions and blood clots
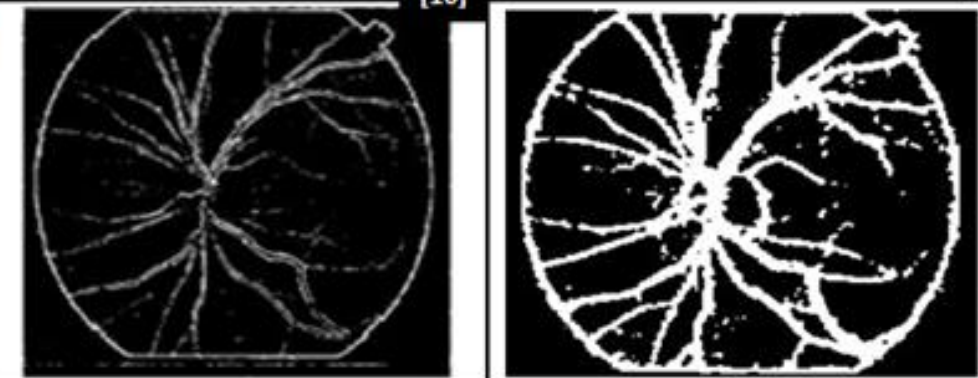

More Prominent Grey Image.
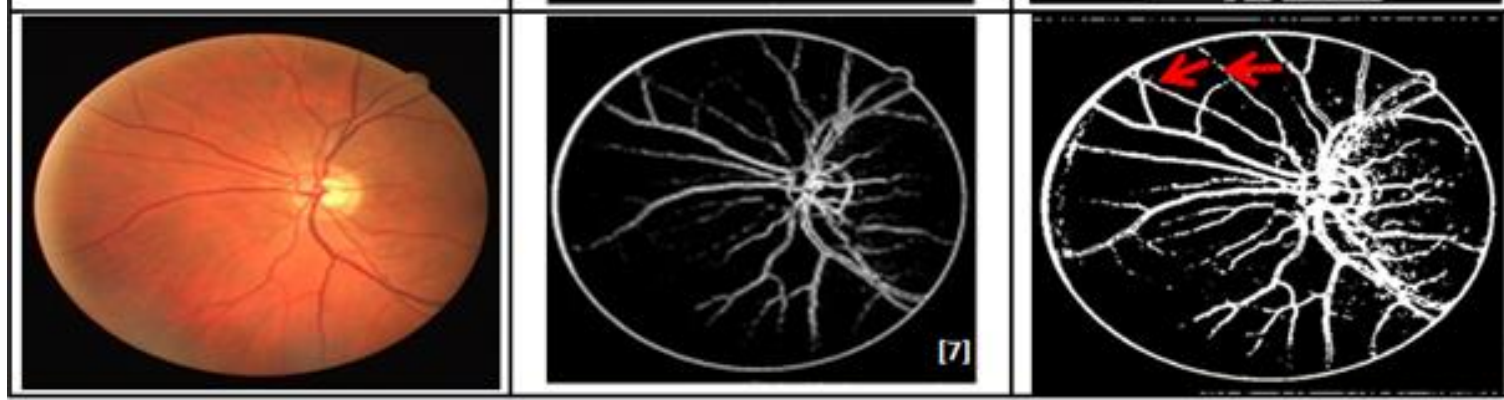

As in, the Extracted Blood

Vessel depicts more continuity and prominence.

Blood Vessel continuity is maintained. 
TABLE II. COMPARISON OF OUR GRAPHICAL USER INTERFACE WITH PRESENT METHODOLOGY FOR DiAgNosis OF RETINAL ABNORMALITIES

\begin{tabular}{|c|c|c|}
\hline Parameters & Fundus Photography + our Graphical User Interface & $\begin{array}{c}\text { Fundus Photography + Optical Coherence } \\
\text { Tomography (OCT) }\end{array}$ \\
\hline Target Audience & $\begin{array}{c}\text { Anyone ( A super specialized Doctor or even a Normal } \\
\text { Person ) }\end{array}$ & Doctor or Experienced Technician \\
\hline Ease of Use & No Expertise required & Expertise required \\
\hline Diagnosis Time & $5-10$ seconds & Few minutes \\
\hline Initial Setup & A Computer/Laptop & The OCT machine \\
\hline Initial Setup Cost & 300 USD (approx.) & 8995 USD (approx.) \\
\hline Cost & NIL & Diagnosis Charge
\end{tabular}

As per Dr.Somnath Das [18], Associate Professor of Regional Institute of Ophthalmology (RIO), Medical College (Kolkata):-"This automated system for the extraction of Retinal blood vessels can give us important clue regarding alteration in morphological pattern and pathological changes in and around the retinal blood vessels, status of laminar blood flow within the blood vessels and nature of extravasations of plasma, blood cells and lipids in surrounding retinal tissue on the basis of which we can diagnose and plan for the management of a large group of ocular diseases. Not only that, this system will also be a good prognostic indicator for a particular disease".

Availability of a Computer/Laptop will enable the user to use the Graphical User Interface after a quick installation of MATLAB. One of the limitations of this Graphical User Interface is that it requires a high processor speed for smoothness and trouble-free output. Other than that, slight lag time can be experienced during the execution of the program.

\section{FutURE PROSPECTS}

As an Initial stage of our work we have chosen Diabetic Retinopathy as our base to validate the Graphical User Interface performance. We can also utilize our Graphical User Interface to understand pathogenesis and ongoing changes in the retinal blood vessels in diseases like Diabetic Retinopathy, Central Retinal Vein Occlusion(CRVO),Branch Retinal Vein Occlusion(BRVO),Central Retinal Artery Obstruction(CRAO),Hypertensive Retinopathy by which we can make staging of the disease, can assess the severity or actual burden of the disease and can make a suitable management protocol to treat it (LASER photocoagulation, intra vitreal injection of anti-Vascular endothelial growth factor etc.). We can also detect and evaluate diseases like HIV Retinopathy, Arterio-venous malfunctions, Optic Atrophy, Ocular diseases like Ocular Ischaemic syndrome, Arteriovenous malformation in carotid-cavernous fistula and haemangioma, Torch infections having an appearance of CMV (Cytomegalovirus) Retinitis, Salt \& Pepper Retinopathy, Rubella etc with the help of our Graphical User Interface. Knowledge about vascular stasis and detection of abnormal optic nerve head perfusion pressure can be extremely helpful as far as the diagnosis of Open angle Glaucoma, Normal tension glaucoma and retinal Vasculitis are concerned. Comparison of structural pattern of retinal vascular tree in Eale's disease, Pan uveitis, Posterior Uveitis and
Takayasu's disease involving retina can be done with this method. Furthermore we can incorporate an algorithm to differentiate between blood clots and lesions and provide a comparative study. This would help in distinguishing various Retinal abnormalities. But for all this we require the fundus photography which is obtained by a fundus Camera which costs almost 3600 USD. To cut down the cost at that level, the next step could be utilizing the Smart Phones' Camera and combining it with an extra lens to capture Fundus images more easily and in a very low cost. This whole setup can then be incorporated in Smart phones as an Application which will be easily accessible and utilized by all.

Further classification of special features detected from the extracted blood vessels along with the use of a trained

Probabilistic Neural Network to recognise and report any of the above mentioned abnormalities can be carried on.

Further classification of special features and parameters detected from the extracted blood vessels along with the use of a trained Probabilistic Neural Network to recognize and report any of the above mentioned abnormalities can be carried out automatically. This Automation of disease detection may also be achieved by the implementation of Neuro-fuzzy template, since it provides a simple way to arrive at a definite conclusion based upon vague, ambiguous, imprecise, noisy, or missing information from the parameters of the pre-processed fundus image for each disease.[19].

\section{CONCLUSIONS}

Retinal images are being used by ophthalmologists to aid in diagnosis, to make measurements, and to look for changes in lesions or severity of diseases. The appearance of the retinal vasculature particularly acts as an indicator for diagnosis of Diabetic Retinopathy (both Proliferative and NonProliferative) and Glaucoma. Therefore, extraction of these features is the key challenge for proper analysis, visualization and quantitative comparison. The present study focuses mainly on this challenge of blood vessel extraction from colour retinal images obtained from fundoscopy. The proposed algorithm proves to be successful and robust in accurately extracting the retinal blood vessels. In this respect, the dataset of 40 test images from the DRIVE database has been used to evaluate this method. Few real time images obtained from the Regional Institute of Ophthalmology (RIO), Medical College, Kolkata are also used for the evaluation. It included fundoscopy of various patients having NPDR (Non 
Proliferative Diabetic Retinopathy), PDR (Proliferative Diabetic Retinopathy) and post PRP (Panretinal Photocoagulation). The fact that the proposed algorithm extracts blood vessels with evident accuracy renders it a quite sought after platform for further improvements. An accurate extraction of blood vessels provides the basis for the measurements of a variety of features including micro aneurysms and hard exudates that can then be applied to the tasks of diagnosis, treatment, evaluation and clinical study [20]. More particularly in rural areas of underdeveloped and developing countries the proposed Graphical User Interface can be utilized to overcome the hardships arising due to the shortage of professional observers by this completely automated computer assisted monitoring and diagnostic system.

\section{ACKNOWLEDGEMENT}

This work was supported by ESL (www.eschoollearning.net), Kolkata.

\section{REFERENCES}

[1] Muthukrishnan, R., Radha, M, (2011), "Edge Detection Techniques for Image Segmentation", International Journal of Computer Science \& Information Technology (IJCSIT), Vol. 3, No.6, pp. 259267,DOI:10.5121/ijcsit.2011.3620.

[2] Yin,Y., Adel, M, Bourennane, S., (2012), "Retinal vessel Segmentation using a Probabilistic Tracking Method", Pattern Recognition, Vol. 45, No. 4, pp. 12335-1244, DOI: 10./1016/j.patcog.2011.09.019.

[3] Zhang, B., Zhang, L., Zhang, L., Karray, F. (2010), "Retinal vessel extraction by matched filter with first-order derivative of Gaussian", Computers in Biology and Medicine, Vol. 40, No. 5, pp. 438445,DOI:10.1016/j.compbiomed.2010.02.008.

[4] Esmaeili, M., Rabbani, H., Mehri, A., Dehghani, A., (2009), "Extraction of retinal blood vessels by curvelet transform", 16th IEEE International Conference on Image Processing (ICIP), pp.33533356, DOI10.1109/ICIP.2009.5413909.

[5] Niemeijer, M., Staal, J., van Ginneken, B., Loog, M., and Abramoff, M., "Comparative study of retinal vessel segmentation methods on a new publicly available database," SPIE Medical Imaging, vol. 5370, pp. 648- 656, 2004

[6] Regional Institute of Ophthalmology (RIO), Medical College (Kolkata).

[7] Karasulu, “Automatic Extraction of Retinal Blood Vessels: A Software Implementation", European Scientific Journal December edition vol.8, No.30 ISSN: 1857 - 7881 (Print) e - ISSN 1857- 7431.

[8] Gao, P., Sun, X., Wang, W., (2010), "Moving Object Detection Based on Kirsch Operator Combined with Optical Flow", International Conference on Image Analysis and Signal Processing (IASP), 9-11 April 2010, pp. 620-624. DOI: 10.1109/IASP.2010.5476045.

[9] Vijayakumari,V. Suriyanarayanan, N., "Survey on the Detection Methods of Blood Vessels in retinal Images", European journal of scientific research ISSN 1450-216X Vol. 68 No.1(2012).

[10] Cemil Kirbas and Francis Quek, "A Review of Vessel Extraction Techniques and Algorithms", ACM computing surveys, Vol. 36, No.2, June 2004.

[11] Raquib Buksh, Soumyajit Routh, Parthib Mitra, Subhajit Banik , Abhishek Mallik, Sauvik Das Gupta "MATLAB based Image Editing and Color Detection", International Journal of Scientific and Research Publications,

[12] Hayashi, J., Kunieda, T., Cole, J., Soga, R., Hatanaka, Y., Lu,M., Hara, T., and Fujita, F., "A development of computer-aided diagnosis system using fundus images", Proc. Of IC on (VSMM 2001), pp. 429-438, 2001.

[13] Global data on visual impairments 2010. Geneva, World Health Organization, 2012.

[14] M.Kavitha, Dr.S.Palani, "A New Fast Curvelet Transform with Morphological Operations based method for Extraction of Retinal blood vessels using Graphical User Interface", International Journal of Scientific \& Engineering Research, Volume 3, Issue 6, June-2012, ISSN 2229-5518.

[15] Archna Sharma and Hempriya, "Detection of Blood Vessels and Diseases in Human Retinal Images", ISSN 2319-7080, International Journal of Computer Science and Communication Engineering IJCSCE Special issue on "Emerging Trends in Engineering \& Management" ICETE 2013

[16] H.S. Bhadauria1, S.S. Bisht and Annapurna Singh, "Vessels Extraction from Retinal Images", IOSR Journal of Electronics and Communication Engineering (IOSR-JECE) e-ISSN: 2278-2834,p- ISSN: 2278-8735. Volume 6, Issue 3 (May. - Jun. 2013), PP 79-82.

[17] David J Browning, , Michael D McOwen, Robert M Bowen, Tisha L O'Marah, "Comparison of the clinical diagnosis of diabetic macular edema with diagnosis by optical coherence tomography", Presented at: American Academy of Ophthalmology Annual Meeting, November, 2003; Anaheim, California.

[18] Dr. Somnath Das, Associate Professor, Regional Institute of Ophthalmology (RIO), Medical College (Kolkata).

[19] Bhijupukan Bhagapathi ChumiDas, "Edge Detection of Digital ImagesUsing Fuzzy Rule Based Technique", International Journal of Advanced Research in Computer Science and Software Engineering, Volume 2, Issue 6, June 2012.)

[20] Osareh, B. Shadgar, "Automatic Blood Vessel Segmentation In Color Images Of Retina", Iranian Journal of Science \& Technology, Transaction B,Engineering, Vol. 33, No. B2, pp 191-206, 2009. 